\title{
El tabaquismo y su fracción atribuible en la enfermedad isquémica cardiaca
}

\author{
Eduardo Salazar-Martínez, Dr en $C_{1}{ }^{(1,2)}$ Luisa María Sánchez-Zamorano, M en $C_{1}{ }^{(1)}$ \\ Luis Alfredo López-Padilla, MD, (2) Adrián Estrada-N ájera, MD, (2) \\ Eduardo C Lazcano-Ponce, Dr en C, ${ }^{(1)}$ Mauricio Hernández-Avila, Ph D.. ${ }^{(1)}$
}

Salazar-Martínez E, Sánchez-Zamorano LM, López-Padilla LA, Estrada-Nájera A,

Lazcano-Ponce EC, Hernández-Avila M. El tabaquismo y su fracción atribuible en la enfermedad isquémia cardiaca. Salud Publica Mex 2002;44 supl 1:S34-S43. El texto completo en inglés de este artículo está disponible en: http://www.insp.mx/salud/index.html

\section{Resumen}

Objetivo. Evaluar el impacto que provoca el tabaquismo so bre la enfermedad isquémica cardiaca (EIC) así como proponer una metodologia simple y eficiente de estimación estándar de la fracción atribuible a éste. Material y métodos. Se realizó un estudio de casos y controles pareado en el Hospital Regional N 0.1 del Instituto Mexicano del Seguro Social de Cuernavaca, estado de Morelos, México, de mayo a junio del año 2000. Los casos fueron 80 pacientes prevalentes e incidentes de EIC. Se seleccio naron aleato riamente 160 controles de la consulta de medicina familiar pareados por edad y sexo. La estimación de la fracción atribuible se obtuvo a través de estimadores sumarios, varianza e intervalos de confianza para diseños pareados por más de un control por cada caso. Resultados La prevalencia de tabaquismo previo al evento isquémico en los casos fue de $80 \%$, comparado con $62 \%$ de prevalencia de los controles. La razón de mo mios de Mantel \& Haenszel mostró una asociación positiva del tabaquismo con EIC (RM, 6.77; IC 95\%, 3.2-13.8). La fracción atribuible entre los expuestos fue de $85 \%$ (IC 95\%, 74-95), mientras que la fracción atribuible poblacional fue de $56 \%$ (IC 95\%, 45-66); en hombres fue de $65 \%$ (IC 95\%,53-77) y en mujeres de 31\% (IC 95\%, 14-47). Conclusiones La fracción atribuible de exposición prolongada al humo del cigarro en EIC en fumadores es de $85 \%$, por esta razón medidas de inter vención primaria para desalentar el consumo de tabaco, y el establecimiento de

\author{
Salazar-Martínez E, Sánchez-Zamorano LM, \\ López-Padilla LA, Estrada-Nájera A, \\ Lazcano-Ponce EC, Hernández-Avila M. \\ Cardiovascular heart disease \\ attributable to smoking. \\ Salud Publica Mex 2002;44 suppl 1:S34-S43. \\ The English version of this paper is available at: \\ http://www.insp.mx/salud/index.html
}

\begin{abstract}
A bstract
Objective. To assess the impact of cigarette smoking on coronary heart disease (CHD) and to propose a simple and efficient method to estimate its attributable fraction (AF). Material and Methods.A matched case-control study was conducted in the Regional Hospital of Instituto Mexicano del Seguro Social (Mexican Institute of Social Security) in Morelos State, Mexico, from May to June-2000. C ases were 80 prevalent and incident patients with CHD. 0 ne-hundredsixty controls matched by sex and age were randomly selected from the outpatient clinic.The attributable fraction was obtained through summary estimators, variances, and confidence intervals for matched designs with more than one control per case. Results The prevalence of cigarette smoking previous to the coronary event was $80 \%$, compared to $62.1 \%$ in controls. Mantel-Haenszel odds ratios showed a positive association of cigarette smoking with CHD (OR, $6.7 ; 95 \% \mathrm{Cl}, 3.2-13.8)$. The attributable fraction among the exposed was $85 \%(95 \% \mathrm{Cl}, 74-95)$ while the population attributable fraction was $56 \%(95 \% \mathrm{Cl}, 45-66)$. The $\mathrm{AF}$ in exposed was $65 \%$ for males $(95 \% \mathrm{Cl}, 53-77)$ and $31 \%$ for females $(95 \% \mathrm{CI} 14-47)$. Conclusions. The long-term smoking AF on CHD is greater than $85 \%$. Such a high AF should be countered with the implementation of primary prevention measures to encourage smoking cessation and with smoking cessation clinics for active smokers. These are the two most important public health interventions to
\end{abstract}

(1) Centro de Investigación en Salud Poblacional, Instituto N acional de Salud Pública. Cuernavaca, Morelos, México.

(2) Instituto Mexicano del Seguro Social, D elegación del estado de Morelos, México.

Fecha de recibido: 31 de mayo de 2001 • Fecha de aprobado: 18 de octubre de 2001 Solicitud de sobretiros: Dr. Eduardo Salazar Martínez. A venida U niversidad 655, Colonia Santa María A huacatitlán, 62508 Cuernavaca, Morelos, México. Instituto N acional de Salud Pública. Centro de Investigación en Salud Poblacional. Dirección de Epidemiología. Correo electrónico: esalazar@ correo.insp.mx 
clínicas antitabaco en fumadores activos, continúan siendo las más relevantes medidas de salud pública actuales, no sólo para prevenir enfermedad cardiovascular, sino un gran espectro de enfermedades crónicas. El texto completo en inglés de este artículo está disponible eh: http:// www.insp. $\mathrm{mx} /$ salud/index.html

Palabras clave: infarto del miocardio; tabaquismo; fracción atribuible; México prevent CHD and a variety of chronic diseases.The English version of this paper is available at: http://www.insp.mx/ salud/index.html

Key words: myocardial infarction; smoking; attributable fraction; Mexico
E 1 tabaquismo es la principal causa de enfermedad, discapacidad y muerte prematura prevenible. Se estima que existen alrededor de 1100 millones de fumadores en el mundo y se proyecta que fallezcan por causas asociadas con el tabaquismo 10 millones de personas para el año 2020; se le atribuyen, asimismo, seis muertes cada minuto. ${ }^{1} \mathrm{El}$ tabaquismo se considera responsable de 36.2 millones de años de vida potencialmente perdidos en el ámbito mundial. ${ }^{2}$ En los Estados Unidos de América (EUA), ocurren 430000 muertes cada año asociadas con el tabaquismo, ${ }^{3}$ y se estima una prevalencia global para ese país de $24.1 \%$, cifras similares a las prevalencias globales reportadas en México (25.8\% en 1988; $25.1 \%$ en 1993 y $27.7 \%$ en 1998); ${ }^{5}$ además, se estiman al menos 40000 muertes asociadas con este hábito cada año en nuestro país, entre las que destacan diversas patologías como cáncer pulmonar, enfermedad isquémica del corazón, enfermedad vascular cerebral y enfermedad pulmonar obstructiva crónica. ${ }^{6}$

A este respecto, las enfermedades cardiovasculares son la principal causa de muerte en los países industrializados, expresadas como cardiopatía isquémica del corazón (EIC); sin embargo, éstas se han incrementado con rapidez en los países en desarrollo, lo que ha provocado que en muchos de ellos se registre también como principal causa de mortalidad. ${ }^{7}$ Los años de vida potencialmente perdidos y atribuidos a muerte por EIC, globalmente ascienden a 46.7 millones. ${ }^{2}$ En países como EUA se registran 481287 defunciones anuales debidas a EIC. ${ }^{8}$ En México representa la principal causa de muerte, con 42516 defunciones registradas en 1997 con una tendencia ascendente desde hace más de 10 años, aun en lo que respecta a morbilidad. ${ }^{9}$

El tabaquismo es ampliamente aceptado como un factor de riesgo para el desarrollo de enfermedades cardiovasculares como resultado directo sobre la hemostasis y la aterosclerosis. ${ }^{10} \mathrm{~A}$ su vez, la aterosclerosis se asocia con exposición a tóxicos del tabaco. ${ }^{11,12}$ El desarrollo de la enfermedad isquémica del corazón debida a tabaquismo se ha descrito mediante cinco procesos que involucran aterosclerosis, trombosis, espasmo arterial coronario, arritmia cardiaca y reducción de la capacidad sanguínea para transportar oxígeno. Los principales efectos se le atribuyen a la nicotina por su acción farmacológica sobre estos mecanismos, y al monóxido de carbono a través de su efecto en el transporte de oxígeno. A otras sustancias como el cadmio, el óxido nítrico y el disulfuro de carbón, también se les ha descrito su participación pero no son claros los mecanismos. ${ }^{13}$ La aterosclerosis es la responsable de la reducción de la luz arterial debido a la proliferación celular en las paredes arteriales, la acumulación de lípidos y, finalmente, la formación de placas y su calcificación (placas ateromatosas). ${ }^{14}$ El tabaquismo tiene un efecto tóxico directo sobre el endotelio. El daño al endotelio tiene un papel importante en el desarrollo de la aterosclerosis, ya que expone a la capa íntima de éste con los lípidos sanguíneos, los leucocitos, y a la agregación plaquetaria. Asimismo, reduce los niveles de lipoproteínas de alta densidad e incrementa las concentraciones de triglicéridos. La trombosis arterial coronaria resulta de la formación de trombo-fibrina plaquetaria, y esto es un elemento clave en la etiología del infarto al miocardio. La agregación plaquetaria incrementada, las concentraciones de fibrinógeno elevadas y las concentraciones de plasminógeno bajas encontradas en sujetos fumadores también se han postulado como un papel importante en la formación de trombosis. El tabaquismo ha mostrado que promueve la vasoconstricción de las arterias coronarias y se ha asociado con un incremento en la prevalencia de $21 \%$ de extrasistolia ventricular prematura. Estas arritmias cardiacas pueden contribuir a una mayor ocurrencia de muerte súbita. ${ }^{15}$

Aunque los factores de riesgo para EIC son ampliamente conocidos en las diferentes regiones del mundo, en nuestro país es limitado el conocimiento del impacto del tabaquismo sobre la EIC; asimismo, no se conoce la fracción atribuible que podríamos 
evitar de EIC en la población general con programas nacionales antitabaco. El objetivo de este estudio es evaluar el impacto que provoca el tabaquismo sobre las EIC, así como proponer una metodología simple y eficiente de estimación estándar de la fracción atribuible (FA).

\section{Material y métodos}

Se realizó un estudio de casos y controles pareados en el Hospital Regional No.1 del Instituto Mexicano del Seguro Social, en la ciudad de Cuernavaca, en el periodo comprendido entre mayo y julio del año 2000. Los casos fueron 80 pacientes de uno u otro sexo tanto prevalentes como incidentes de enfermedad isquémica cardiaca, con diagnóstico clínico y confirmación electrocardiográfica o enzimática, con edades entre los 38 y 80 años de edad, provenientes tanto de la consulta externa de cardiología como del servicio de urgencias del hospital, quienes acudieron a su consulta de control para los casos prevalentes, o como evento agudo no fatal por primera vez en los casos incidentes. Los controles fueron obtenidos aleatoriamente de la consulta externa de medicina familiar del mismo hospital donde se obtuvieron los casos. Se seleccionaron dos controles por cada caso, pareados por sexo y por grupos de frecuencia de edad ( \pm 2 años) hasta completar 160 controles totales. Los motivos más frecuentes de consulta en los controles fueron padecimientos respiratorios, gastrointestinales, oftalmológicos, del sistema musculoesquelético y dermatológicos, entre otros, los cuales no incluyeron control de enfermedades crónicas ni sospecha o diagnóstico de enfermedad pulmonar obstructiva crónica, cáncer pulmonar, tuberculosis, asma, bronquitis y enfisema. Todos los participantes en el estudio tuvieron una residencia mínima de un año en la ciudad de Cuernavaca, garantizando así la cobertura y acceso al servicio.

La definición de la exposición se realizó de forma que se estimara el tabaquismo en igualdad de tiempo, tanto en los controles como en los casos (ya que la mayoría de los casos son prevalentes, esto es, son sobrevivientes al evento isquémico).

Se agrupó a la población de estudio en dos categorías: a) el grupo de individuos que nunca habían fumado o que tenían 15 años o más de haber dejado de fumar respecto a la edad de ocurrencia del evento en los casos, y b) el grupo de individuos que reportaron fumar activamente hasta el momento de ocurrencia del evento en el grupo de casos, o que fueran ex fumadores con menos de 15 años de haber dejado de fumar. El $95 \%$ de la población de estudio ha fumado por más de 10 años. De los fumadores activos, el tiempo mínimo de consumo de tabaco en forma continua fue mayor de 15 años (rango de 15.5 a 63 años). Se construyó, asimismo, una variable de intensidad de tabaquismo basada en el consumo anual de cigarrillos, multiplicándola por los años de ser fumadores; para efecto del análisis, en esta variable se formaron cuatro categorías: a) no fumadores, b) fumadores de bajo consumo ( $\leq 66570$ cigarrillos), c) fumadores de mediano consumo (66571 a 165600 ) y d) fumadores de alto consumo (>165 600).

El cálculo del tamaño muestral requerido para estimar fracción atribuible se realizó con un $\alpha=0.05 \mathrm{y}$ una $\beta=0.20$ para una relación caso/control de 1:2, mediante la fórmula descrita por Browner y Newman ${ }^{16}$ (anexo I). El cálculo de tamaño de muestra fue estimado con base en la exposición a tabaco, con una prevalencia esperada de al menos $50 \%$.

Se utilizaron dos cuestionarios diseñados específicamente para el estudio, uno para obtener información sobre características sociodemográficas, antecedentes patológicos, antropometría, estilos de vida y consumo de tabaco, y otro para obtener información clínica, complementada con los registros médicos. Todos los participantes aceptaron formar parte del estudio mediante un formato de consentimiento informado.

Se realizó un análisis de los datos que incluyó explorar el comportamiento de las variables, así como obtener información sobre variables asociadas a EIC mediante un análisis bivariado y multivariado a través de modelos de regresión logística condicionada múltiple, utilizando el paquete estadístico STATA-V6.

Adicionalmente, en los casos se utilizó un análisis de supervivencia para evaluar la velocidad de presentación del evento (EIC) en sujetos fumadores y no fumadores. La estimación de las curvas de sobrevida en ambos grupos estuvo basada en la definición de exposición a tabaquismo propuesta previamente.

La estimación de la fracción atribuible se realizó mediante la metodología propuesta por Kuritz y Landis. ${ }^{17}$ Dicha propuesta nos permitió obtener estimadores, varianzas e intervalos de confianza al $95 \%$ para fracción atribuible de estudios de casos y controles pareados por más de un individuo por cada caso, realizándose posteriormente un análisis estratificado por sexo. El principio de este método se conceptualiza como un simple muestreo aleatorio de casos equivalente a un muestreo aleatorio de sus controles pareados. Esto permite cruzar la información en los estratos formados por los grupos de pares combinando los datos, aproximándose al procedimiento de MantelHaenszel, para estimar la fracción atribuible entre los expuestos y entre la población general (anexo II). Asi- 
mismo, para evaluar la FA por intensidad de consumo, se utilizó el efecto independiente del tabaquismo asociado con EIC, cuyo estimador se ajustó por ingreso quincenal.

\section{Resultados}

En los 80 casos de EIC, la localización más frecuente de la isquemia fue la inferior $(32.5 \%)$, seguida de la antero-septal $(17.5 \%)$, la infero-septal $(16.3 \%)$, la postero-inferior y la anterior extensa (6.2\% cada una); la anterior, inferior con antero-septal y la diafragmática (5\% cada una), la antero-inferior (3.8\%) y la septal sin onda Q (2.5\%). El cuadro I muestra las características generales de la población en el estudio. La prevalencia de hipertensión arterial fue mayor en los casos al compararla con los controles (61.3\% vs $28.1 \%$ ). De igual manera, existe una mayor prevalencia de hipercolesterolemia en los casos ( $43.8 \%$ vs $13.1 \%$ en los controles); mientras que la prevalencia de diabetes mellitus se duplica en los casos (32.5\% vs 16.9\%) (datos no mostrados en cuadros).

No se encontraron diferencias de edad entre los casos (media: $61.9 \pm 8.75$ años) y los controles (media: $61.6 \pm 8.51$ años) debido al pareamiento. De manera general, el porcentaje de mujeres en el estudio fue de $23.8 \%$, mientras que el de hombres fue de $76.2 \%$; sin embargo, al evaluar por caso y control los grupos fueron idénticos como efecto del pareamiento (cuadro I). La mayoría de los individuos de ambos grupos convive con una pareja. En cuanto a la ocupación de los casos, la mitad de la población fue de jubilados (51.3\%) en comparación con el grupo control, en donde el mayor porcentaje se concentró en personas laboralmente activas (41.2\%). Los años de estudio se distribuyeron de la siguiente manera: en el grupo de los casos $45 \%$ realizó estudios durante cinco años o menos, mientras que el grupo control fue similar con $53.1 \%$. La media de años de escolaridad en los casos fue de $7.2 \pm 5.75$ años, mientras que los controles tuvieron una media de estudios menor (5.4 \pm 5.09 años, $p=0.01$; datos no mostrados en cuadros). En cuanto a ingreso económico, el mayor porcentaje de ingreso en cada grupo correspondió a la categoría de ingreso quincenal mayor de $\$ 1550.00$. De manera similar a la escolaridad, el ingreso quincenal fue mayor en los casos con una media de ingreso de $\$ 2742.00$ pesos y para los controles de $\$ 1569.00$ pesos, de manera significativa $(p<0.01$; datos no mostrados en cuadros).

El 80\% de los casos reportó haber fumado alguna vez en su vida, en contraste con $61.8 \%$ de los controles. Como categoría de fumador actual, en el grupo de los casos sólo se registró una prevalencia de 7.5\%, 20\%

\section{Cuadro I \\ Características sociodemográficas \\ DE LA POBLACIÓN DE ESTUDIO. \\ Instituto Mexicano del Seguro Social. Cuernavaca, Morelos, México, 2000}

Factor $\quad \frac{\text { Casos }}{\text { No. }(\%)} \quad \frac{\text { Controles }}{\text { No. }(\%)} \quad$ Valor de p*

Edad (años) $)^{\ddagger}$

\begin{tabular}{llllll}
$38-56$ & 17 & $(21.2)$ & 42 & $(26.2)$ & \\
\hline $57-63$ & 22 & $(27.5)$ & 42 & $(26.2)$ & \\
\hline $64-67$ & 26 & $(32.5)$ & 38 & $(23.8)$ & \\
\hline $68-80$ & 15 & $(18.8)$ & 38 & $(23.8)$ & 0.43
\end{tabular}

$\operatorname{Sex} 0^{\ddagger}$

\begin{tabular}{lllrll} 
Mujer & 19 & $(23.8)$ & 38 & $(23.8)$ & \\
\hline Hombre & 76 & $(76.2)$ & 122 & $(76.2)$ & 1.00
\end{tabular}

Estado civil

Soltero/divorciado/viudo $\quad 19 \quad$ (23.8) $30 \quad$ (18.8)

\begin{tabular}{llllll}
\hline Casado/unión libre & 61 & $(76.2)$ & 130 & $(81.3)$ & 0.36
\end{tabular}

0 cupación

\begin{tabular}{lrrrrl} 
Hogar & 14 & $(17.5)$ & 30 & $(18.8)$ & \\
\hline Profesional & 8 & $(10.0)$ & 4 & $(2.5)$ & \\
\hline Empleado/obrero & 17 & $(21.2)$ & 66 & $(41.2)$ & \\
\hline Jubilado & 41 & $(51.3)$ & 60 & $(37.5)$ & 0.002
\end{tabular}

Escolaridad (años)

\begin{tabular}{llllll}
$\leq 5$ & 36 & $(45.0)$ & 85 & $(53.1)$ & \\
\hline $6-11$ & 23 & $(28.8)$ & 51 & $(31.9)$ & \\
\hline$>11$ & 21 & $(26.2)$ & 24 & $(15.0)$ & 0.10
\end{tabular}

Ingreso quincenal (\$)

\begin{tabular}{llllll}
$\leq 1150$ & 13 & $(17.1)$ & 49 & $(31.2)$ & \\
\hline $1151-1550$ & 32 & $(42.1)$ & 50 & $(31.9)$ & \\
\hline$>1550$ & 31 & $(40.8)$ & 58 & $(36.9)$ & 0.06
\end{tabular}

* Prueba de ji cuadrada

₹ Variables de pareamiento

como nunca fumador y $72.5 \%$ como ex fumador, esto como efecto de ser casos prevalentes. En el grupo control, $21.9 \%$ es fumador actual, $38.1 \%$ no fuma y $21.9 \%$ es ex fumador.

La intensidad del tabaquismo muestra una prevalencia mayor en los casos al compararla con los controles, tanto por número total de cigarrillos fumados, como por cajetillas comparadas anualmente. La edad de inicio del tabaquismo fue similar en ambos grupos, con una media de 16 años estimada por el método de Ka- 
plan-Meier. En la figura 1 podemos observar las curvas de tiempo al evento isquémico. Desde el inicio del tabaquismo hasta el primer evento isquémico en los casos, la mediana de aparición del evento para los fumadores fue 45 años posterior al inicio del tabaquismo, mientras que para los no fumadores, la mediana fue 55 años después de este inicio, esto es, los fumadores desarrollaron el evento, en promedio 10 años antes que los no fumadores, de manera significativa (" $p$ " de LogRank= 0.000).

Respecto del abandono del hábito, los casos tuvieron una media de 10.9 años, y los controles de 15.06 años ( $p=0.04$ ). En cuanto al consumo de alcohol, podemos observar un patrón de consumo heterogéneo, bebedores actuales son más prevalentes en los controles ( $22.5 \%$ vs $15.0 \%)$, pero los ex bebedores son más prevalentes en los casos (68.7\% vs 53.7\%) (cuadro II).

La hipertensión arterial mostró un riesgo cuatro veces mayor de padecer EIC (RM, 4.0; IC 95\%, 2.1-7.4), la hipercolesterolemia tuvo un riesgo similar $(R M, 4.3$; IC 95\%, 2.1-8.7) y la diabetes mellitus triplicó el riesgo de EIC (RM, 2.9; IC 95\%, 1.4-5.9). La intensidad del tabaquismo mostró un riesgo cinco veces mayor de desarrollar EIC al comparar la categoría de alta intensidad con los no fumadores, con una prueba de tendencia altamente significativa $(R M, 5.3$; IC95\%, 2.2-12.9; $p$ de tendencia $=0.003$ ) (figura 2). Las fracciones poblacionales atribuidas a tabaquismo por categoría de intensidad fueron las siguientes: a) baja intensidad, $36 \%$; b) mediana intensidad, $31 \%$ y c) alta intensidad, $49 \%$. El consumo de alcohol no se asoció con la EIC.

En relación con el riesgo atribuible del tabaquismo en la EIC, la prevalencia de acuerdo con el tiempo de abandono en los casos fue de $66.2 \%$, mientras que

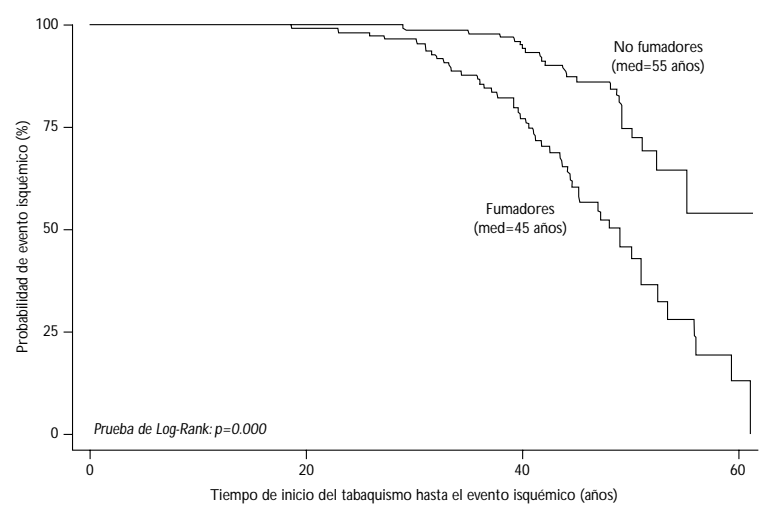

Figura 1. Comparación de tiempo transcurrido para la presencia de Enfermedad Isquémica Cardiaca en FUMADORES Y NO FUMADORES. INSTITUTO MEXICANO DEL Seguro Social, Cuernavaca, Morelos, México, 2000

\section{Cuadro II}

Patrón de consumo de tabaco en la población de estudio. Instituto Mexicano del Seguro Social. Cuernavaca, Morelos, México, 2000

Factor $\quad \frac{\text { Casos }}{\text { No. }} \quad \frac{\text { Controles }}{\text { No. }} \quad(\%) \quad$ Valor de p*

Antecedente de tabaquismo ${ }^{\ddagger}$

\begin{tabular}{llllll} 
No & 16 & $(20.0)$ & 61 & $(32.1)$ & \\
\hline Sí & 64 & $(80.0)$ & 99 & $(61.8)$ & 0.005
\end{tabular}

Categoría de fumador ${ }^{\ddagger}$

\begin{tabular}{llllll} 
N unca & 16 & $(20.0)$ & 61 & $(38.1)$ & \\
\hline Actual & 11 & $(13.7)$ & 52 & $(32.5)$ & \\
\hline Ex fumador & 53 & $(66.3)$ & 47 & $(29.4)$ & 0.00
\end{tabular}

Tabaquismo según tiempo de abandono del hábito

\begin{tabular}{lllrll}
$\geq 15$ años $^{5}$ & 27 & $(33.8)$ & 106 & $(66.2)$ & \\
\hline$<15$ años $^{\#}$ & 53 & $(66.2)$ & 54 & $(33.8)$ & 0.00
\end{tabular}

Intensidad del tabaquismo

\begin{tabular}{llllll} 
No fumadores & 16 & $(20.0)$ & 61 & $(38.1)$ & \\
\hline Bajo & 25 & $(31.3)$ & 57 & $(35.6)$ & \\
\hline Medio & 14 & $(17.5)$ & 22 & $(13.8)$ & \\
\hline Alto & 25 & $(31.2)$ & 20 & $(12.5)$ & 0.001
\end{tabular}

Cigarros consumidos diariamente

\begin{tabular}{lrrrrr} 
No fumador & 16 & $(20.0)$ & 61 & $(38.1)$ & \\
\hline$\leq 20$ & 54 & $(67.5)$ & 95 & $(59.4)$ & \\
\hline$>20$ & 10 & $(12.5)$ & 4 & $(2.5)$ & 0.001
\end{tabular}

$N$ o. de cajetillas consumidas al año

\begin{tabular}{llllll} 
No fumador & 16 & $(20.0)$ & 61 & $(38.1)$ & \\
\hline$\leq 54$ & 24 & $(30.0)$ & 50 & $(31.2)$ & \\
\hline$>54$ & 40 & $(50.0)$ & 49 & $(30.6)$ & 0.004
\end{tabular}

Consumo de alcohol

\begin{tabular}{llllll} 
N unca & 13 & $(16.25)$ & 38 & $(23.75)$ & \\
\hline Ex bebedores & 55 & $(68.75)$ & 86 & $(53.75)$ & \\
\hline Actualmente & 12 & $(15.0)$ & 36 & $(22.5)$ & 0.08 \\
* Prueba de ji cuadrada & & & & & \\
₹ Al evento isquémico en los casos \\
§ Esta categoría incluye a sujetos que nunca han fumado \\
* Incluye a los fumadores activos
\end{tabular}

en los controles fue de 33.8\%, la razón de momios de Mantel \& Haenszel $\left(R M_{\mathrm{MH}}\right)$ mostró una asociación positiva con el tabaquismo, esto es, el haber dejado de fumar hace menos de 15 años o ser fumador activo incrementa 6.7 veces el riesgo de EIC (IC95\%, 3.2-13.8). La fracción atribuible en los expuestos fue de $85 \%$ (IC $95 \%, 74-95)$, mientras que la fracción atribuible po- 


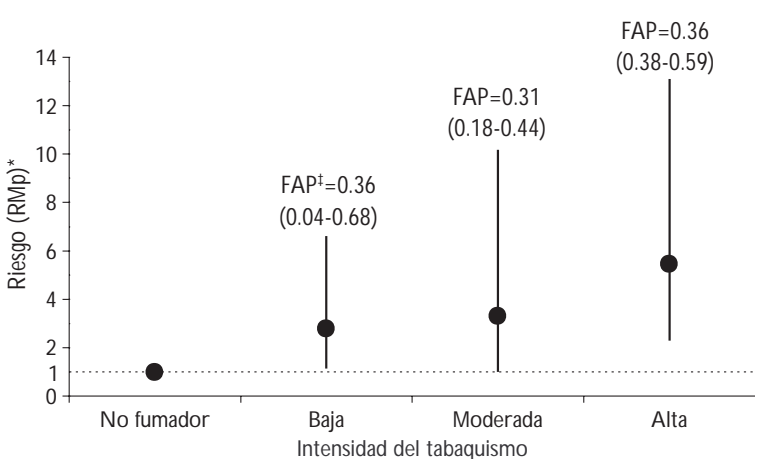

* Razón de momios pareada por edad y sexo, ajustado por ingreso quincenal

₹ Fracción atribuible poblacional con IC 95\%

Figura 2. Riesgo de Enfermedad Isquémica Cardiaca CON RELACIÓN A LA INTENSIDAD DEL TABAQUISMO Y EN SU fraccion atribuible. Instituto Mexicano del Seguro Social, Cuernavaca, Morelos, México, 2000

blacional fue de $56 \%$ (IC 95\%, 45-66). Respecto del sexo, se observó que el riesgo en hombres y mujeres es similar, $R M_{\mathrm{MH}}=7.0(\mathrm{IC} 95 \%, 3.1-15.8)$ y $R M_{\mathrm{MH}}=6.0$ (IC $95 \%, 1.2-28.7)$, respectivamente, al igual que la fracción atribuible en los expuestos, siendo en hombres de $85.7 \%$ (IC 95\%, 73.9-97.4) y en mujeres de $83.3 \%$ (IC 95\%, 56.9-99.4); sin embargo, la fracción atribuible poblacional presenta mayor contribución del tabaquismo en EIC en los hombres: $\mathrm{FAP}=64.6 \%$ (IC 95\%, 52.6-76.7), que en las mujeres: $\mathrm{FAP}=30.7 \%$ (IC 95\%, 14.2-47.2).

\section{Discusión}

Los resultados del presente estudio muestran la gran importancia que tiene evaluar el impacto del tabaquismo en la EIC y ampliar el panorama para otras enfermedades que están relacionadas con el consumo del tabaco. En otros países, el riesgo atribuible del tabaquismo en la EIC ya ha sido evaluado, en la mayoría de ellos, para observar su impacto en la mortalidad. El cuadro III muestra los principales estudios que han reportado FA y la forma en que definieron la exposición. Estudios realizados en los EUA, reportan una FA de $48 \%$ en mujeres de Harlem, mientras que en los hombres del mismo lugar es de $52 \%$. En Nueva York muestran una FA similar en ambos sexos (37\% y $38.5 \%$, respectivamente), y globalmente en ese país se reporta una FA de 37\% para las mujeres y de $45 \%$ en los hombres. Los autores definieron a los fumadores como aquellos individuos que habían fumado uno o más cigarrillos en el día en el último mes, y si en su vida habían fumado más de 100 cigarrillos. ${ }^{18}$ En Canadá, el Nutrition Canada Survey obtuvo una FA de $47 \%$ en los hombres y de $10 \%$ en las mujeres, siendo esta fracción mayor en edades menores de 65 años en contraste con la población de mayor edad, los fumadores fueron definidos de acuerdo con consumo de tabaco alguna vez en su vida. ${ }^{19}$ Otro análisis en el mismo país que abarca toda la provincia de Québec, reporta una FA de 39\% en menores de 65 años y solamente de $18.5 \%$ en mayores de esa edad; los fumadores fueron definidos de acuerdo con su estado actual de fumador al momento de la entrevista. ${ }^{20}$ En nuestro es-

Cuadro III

Principales CaRacterísticas y definición de La VARIAble de eXposición en estudios QUE REPORTAN FRACCIÓN ATRIBUIBLE POBLACIONAL DEL TABAQUISMO EN EIC. Instituto Mexicano del Seguro Social. Cuernavaca, Morelos, México, 2000

\begin{tabular}{|c|c|c|c|c|}
\hline Referencia & Tipo de estudio & $\begin{array}{l}\text { Operaci } \\
\text { variable }\end{array}$ & $\begin{array}{l}\text { nalización de la } \\
\text { de tabaquismo }\end{array}$ & Resultados \\
\hline $\begin{array}{l}\text { Semenciw y } \\
\text { colaboradores } 1988\end{array}$ & Cohorte (Mortalidad por EIC) & $\begin{array}{l}\text { Fumadores actuales } \\
\text { Ex fumadores }\end{array}$ & $\begin{array}{l}\text { Tabaquismo al momento del estudio } \\
\text { Sin tabaquismo al momento del estudio }\end{array}$ & $\begin{array}{l}\mathrm{FA} \\
\mathrm{FA}_{\text {mujeres }(65-79 \text { años })}=3 \% \\
\text { mujeres (35-64 años) }\end{array}$ \\
\hline $\begin{array}{l}\text { Levesque y } \\
\text { colaboradores } 1998\end{array}$ & Mortalidad & $\begin{array}{l}\text { No fumadores (nunca) } \\
\text { Fumador } \\
\text { Ex fumador } \\
\text { No fumador }\end{array}$ & $\begin{array}{l}\text { N unca fumaron } \\
\text { Tabaquismo activo y }+100 \text { cigarros/vida } \\
\text { Sin tabaquismo activo }+100 \text { cigarros/vida } \\
N \text { unca fumaron }\end{array}$ & $\begin{array}{l}F A<65 \text { años }=39 \% \\
F A \geq 65 \text { años }=18.5 \%\end{array}$ \\
\hline $\begin{array}{l}\text { Northridge y } \\
\text { colaboradores } 1998\end{array}$ & Mortalidad & $\begin{array}{l}\text { Fumador } \\
\text { No fumador }\end{array}$ & $\begin{array}{l}\text { Fumadores aquellos individuos que habían fumado uno o más } \\
\text { cigarrillos en el día en el último mes y + } 100 \text { cigarrillos/vida } \\
N \text { unca fumaron }\end{array}$ & $\begin{array}{l}\mathrm{FA}_{\text {hombres }}=45 \% \\
\mathrm{FA}_{\text {mujeres }}=37 \%\end{array}$ \\
\hline $\begin{array}{l}\text { Salazar y } \\
\text { colaboradores } 2000\end{array}$ & Casos y controles & $\begin{array}{l}\text { Fumador } \\
\text { No fumador }\end{array}$ & $\begin{array}{l}\text { Fumador activo + aquel ex fumador que tenía menos } \\
\text { de } 15 \text { años de abandono del tabaquismo } \\
\text { No fumador + ex fumador con } 15 \\
\text { años o más de abandono del tabaquismo }\end{array}$ & $\begin{array}{l}\mathrm{FA}_{\text {general }}=56 \% \\
\mathrm{FA}_{\text {mujeres }}=30.7 \% \\
\mathrm{FA}_{\text {hombres }}=64.6 \%\end{array}$ \\
\hline
\end{tabular}


tudio encontramos una FAP mayor a las reportadas previamente aun controlando la edad y el sexo, sin embargo, coincide respecto de los datos obtenidos para hombres, en los cuales la FAP es más elevada que en las mujeres.

La metodología empleada en este estudio, además de realizar una estimación eficiente, ya que se requiere de menor costo y una búsqueda de sujetos en menor tiempo para realizar los cálculos, permite ponderar la fracción atribuible correspondiente al tabaquismo en la mortalidad por EIC. Para 1998, en México se registraron 42683 defunciones por enfermedad isquémica del corazón, de las cuales se estima que 23902 son atribuidas al tabaquismo. Esta estrategia permitirá la evaluación de las intervenciones y actividades de los programas antitabaco y probablemente tendrá capacidad para detectar cambios en los hábitos de los fumadores, tal como el abandono del tabaquismo, principalmente en las poblaciones jóvenes que inician el uso del tabaco, ya que se ha reportado que el cese en el tabaquismo no conlleva beneficios inmediatos en la salud, sino hasta pasados 15 años después de abandonar su consumo. ${ }^{21}$

La prevalencia de exposición a tabaquismo en el estudio de Morelos es comparable con los estudios realizados en otros países porque, en más de $80 \%$ de los casos, el tabaquismo activo se presentó por un periodo mayor de 10 años e incluso, con la definición propuesta por los autores, esta exposición se encuentra subestimada. En los controles, la prevalencia de tabaquismo fue de $61.8 \%$, similar a la informada por grupo de edad en la Encuesta Nacional de Adicciones $1993 .{ }^{22}$ Es necesario puntualizar las grandes limitaciones que tienen estudios que utilizan una definición de exposición a tabaquismo de al menos 100 cigarrillos en el periodo de vida, en un evento con un largo periodo de latencia.

Otro aspecto importante es la utilidad del estudio en cuanto a las características de la población. Interesantemente, a pesar de tratarse de una población con características homogéneas, los casos mostraron tener mayor ingreso y escolaridad que los controles, y concordaron estos resultados con otros estudios. Esto permite orientar a los programas antitabaco para que incidan en la población más susceptible, mediante intervenciones educativas en la población joven $(80.5 \%$ de la población fumadora se encuentra en niveles educativos de primaria, secundaria y preparatoria), ${ }^{22}$ y la posibilidad de gravar un impuesto al tabaco.

Una de las limitantes más importantes del estudio consiste en la introducción potencial de un sesgo de selección al utilizar conjuntamente casos prevalentes $(\mathrm{n}=64)$ y casos incidentes $(\mathrm{n}=16)$; sin embargo, realizamos un análisis estratificado por tipo de caso, y obtu- vimos FAP similares en ambos grupos (57\% para casos incidentes y $56 \%$ para prevalentes), lo que nos permite sugerir que la FAP no se afecta por este hecho. Asimismo, el efecto del sesgo de selección originado por el estudio de casos prevalentes depende de la relación entre tabaquismo y la probabilidad de muerte por infarto. Por otro lado, al utilizar casos prevalentes se puede potencialmente introducir un sesgo de selección que subestima el efecto del tabaco en la EIC (ya que no se están incluyendo a los que murieron por el evento).

Los estudios de casos y controles usados comúnmente para estudiar relaciones causales a través de estimadores de la tasa de incidencia, como la razón de momios, no permiten observar el impacto de la relación encontrada en la población blanco; ésta se realiza a través de la fracción atribuible. Diversos autores han contribuido con metodologías para su estimación, sin embargo, la presente estrategia permite eliminar algunos factores confusores desde el diseño ya que posibilita parear a los casos con diversos números de controles, ofreciendo además el cálculo directo de estimadores sumarios, varianzas e intervalos de confianza para la fracción atribuible entre los expuestos, así como en la fracción atribuible poblacional.

En conclusión, la fracción atribuible del tabaquismo sobre la EIC es elevada. La metodología empleada nos permite evaluar eficientemente la fracción atribuible y ponderar su participación en la mortalidad por esta patología, y tal vez detectar cambios derivados de las actividades de los programas antitabaco.

\section{Referencias}

1. W orld Health O rganization. The W orld Health Report 1995. Bridging the gaps. Ginebra:W HO 1995.

2.Murray CJL, LópezAD. $0 \mathrm{n}$ the comparable quantification of health risks: Lessons from the global burden of disease study. Epidemiology 1999;10: 594-605.

3. Centers for D isease Control and Prevention (CDC). Smoking attributable mortality and years of potential life lost - United States, 1984. MMW R Morb Mortal W kly Rep 1997; 46: 444-451.

4. Centers for D isease Control and Prevention (CDC). Cigarette smoking among adults - United States, 1998. MMW R Morb Mortal W kly Rep 2000;49:881-884.

5. Tapia-C onyer R, Cravioto P, Medina-Mora ME, Hoy MJ, Kuri P. El consumo de tabaco en México. Encuestas N acionales de Adicciones 1988, 1993, 1998. México, D.F.: Secretaría de Salud, 2000: 53-60.

6. Centers for D isease Control and Prevention (CDC). Smoking-Attributable mortality mortality-Mexico, 1992. MMW R Morb Mortal W kly Rep 1995; 44(19): 372-373, 379-381.

7.W orld Health 0 rganization,W orld health statistical annual: 1996. G inebra: W HO, 1998.

8.American Heart Association. 1998 Heart and stroke statistical update. Dallas:AHA, 1998. 
9. N úcleo de acopio y análisis de información en salud (N AAIS). Instituto $\mathrm{N}$ acional de Salud Pública, México 2001. D isponible en:http://naais.insp.mx/ consulta/ mortalidad.

10. US O ffice on Smoking and Health. The health consequences of smoking: Cardiovascular diseases: A report of the Surgeon General. W ashington, DC: US G overnment Printing 0 ffice, 1989: 179-203.

11. Howard G, Burke GL, Szklo M, Tell GS, Eckfeldt J, Evans G et al.Active and passive smoking are associated with increased carotid wall tickness: The atherosclerosis risk in communities study. Arch Intern Med 1994; 154: 1277-1282.

12. Diez-Roux AV, N ieto FJ, Comstock GW, H oward G, Szklo M.The relationship of active and passive smoking to carotid atherosclerosis 12-14 years later. Prev Med 1995; 24: 48-55.

13. US D epartment of health and human services. The health benefits of smoking cessation.A report of the Surgeon General.W ashington, DC .:US Government Printing 0 ffice, 1990.

14. Munro JM, Cotran RS. The pathogenesis of Arterosclerosis: Atherogenesis and inflammation. Lab Invest 1988; 58:249-261.
15. Hennekens CH, Lown B, Rosner B, Grufferman S, Dalen J. Ventricular premature beats and coronary risk factors. Am J Epidemiol 1980;112:93-99. 16. Browner W S, N ew man TB. Sample size and power on the population attributable fraction. Am J Public Health 1989; 79: 1289-1294.

17. Kuritz SJ, Landis JR. Attributable risk estimation from matched casecontrol data. Biometrics 1988; 44: 355-367.

18. N ortridge ME, Morabia A, Ganz ML, Basset MT, Gemson D, Andrews $\mathrm{H}$ et al. Contribution of smoking to excess mortality in harlem. Am J Epidemiol 1998; 147: 250-258.

19. Semenciw RM, Morrison HI, Mao Y, Johansen H, Davies JW, W igle DT. Major risk factors for cardiovascular disease mortality in adults: Results from the nutrition Canada survey cohort. Int J Epidemiol 1988;17:317-324. 20. Lévesque B, Rochette L, Gingras S. Mortalité attributable au tabagisme au Q uébec. C an J Public Health 1998; 89: 28-32.

21. Enstrom JE, Heath CW Jr. Smoking cessation and mortality trends among 118,000 Californians, 1960-1997. Epidemiology 1999; 10: 500-512. 22. Secretaría de Salud. Encuesta N acional de Adicciones 1993. Tabaco. México, D.F.: Dirección General de Epidemiología, SSA. 1994. 
Anexo I

Cálculo del tamaño muestral para diseños de casos y controles pareados

El número de casos mínimo necesario para que un estudio con un poder de $80 \%(1-b=0.20)$, con un nivel de confianza de $95 \%(a=0.025)$ en una prueba de una cola; con una prevalencia variable de la exposición en el grupo control y con una relación de 1:2 entre casos y controles, buscando determinar diferentes fracciones atribuibles poblacionales, se determina utilizando la fórmula descrita por Browner y N ewman.16

$$
c^{\prime}=\frac{\left[Z_{\alpha} \div(1+1 / b) P(1-P)+Z_{\beta} \div p_{c}\left(1-p_{c}\right)+p_{x}\left(1-p_{x}\right) / b\right]^{2}}{\left(p_{c}-p_{x}\right)^{2}}
$$

donde:

$Z_{\alpha}=1.96$

$Z_{\beta}^{\alpha}=1.645$

$p_{x}=$ Proporción de la exposición en los controles

$p_{c}=$ Proporción de la exposición en los casos

$\mathrm{P}=$ Proporción completa de sujetos (casos y controles) expuestos

$b=$ Razón de controles $/$ casos $(2: 1)$

Donde:

$$
\begin{aligned}
& p_{c}=F A P+p_{x}(1-F A P) \\
& P=\frac{p c+b p x}{b+1}
\end{aligned}
$$

$\mathrm{FAP}=$ Fracción atribuible poblacional

El número correcto de casos

$$
c=c^{\prime}+\frac{b+1}{b\left|p_{c}-p_{x}\right|}
$$




\section{Anexo II \\ ESTIMACIÓN DE LA FRACCIÓN ATRIBUIBLE PARA UN ESTUDIO DE CASOS Y CONTROLES PAREADO POR MÁS DE UN CONTROL POR CADA CASO}

La descripción de los datos se realiza de acuerdo con la variable de tabaquismo construida a partir de los siguientes criterios: a) como categoría de referencia, aquellos sujetos que nunca fumaron + aquellos que dejaron de fumar hace más de 15 años; b) como categoría de exposición a sujetos que fuman activamente + aquellos que tienen menos de 15 años de haber dejado de fumar. Se utiliza la metodología propuesta por Kuritz \& Landis ${ }^{18}$ para calcular la Fracción Atribuible en estudios pareados 1:k.

Combinaciones posibles de parejas de casos y controles pareados 1:2

Patrón de respuesta

1
2
3
4
5
6

Casos expuestos ( $\mathrm{x})$

$$
\begin{aligned}
& 1 \\
& 1 \\
& 1 \\
& 0 \\
& 0 \\
& 0
\end{aligned}
$$

Controles expuestos (y)
Total de controles

$\begin{array}{ll}2 & \mathrm{~L}_{102}=19 \\ 2 & \mathrm{~L}_{112}=23 \\ 2 & \mathrm{~L}_{122}=11 \\ 2 & \mathrm{~L}_{002}=18 \\ 2 & \mathrm{~L}_{012}=9 \\ 2 & \mathrm{~L}_{022}=0\end{array}$

El patrón de respuesta significa la relación que existe entre los casos con sus respectivos controles respecto de la variable de exposición. En este caso en particular, los primeros tres patrones se refieren a los casos expuestos; donde el 1 son el número de casos expuestos y sus dos controles no expuestos; el 2 se refiere al número de casos expuestos, un control no expuesto; y el 3 al número de casos expuestos y los dos controles expuestos. Los tres últimos patrones se refieren a los casos no expuestos donde el 4 es el número de casos no expuestos y sus dos controles no expuestos; el 5 al total de casos no expuesto y con un control no expuesto; y el 6 los casos no expuestos con sus dos controles expuestos.

\begin{tabular}{|c|c|c|c|c|}
\hline Patrón de respuesta & $\mathrm{L}_{1 y \mathrm{k}}$ & A & $A L_{1 y k}$ & $(A)^{2} \mathrm{~L}_{1 y k}$ \\
\hline $\begin{array}{c}1 \\
2 \\
3 \\
\text { Total }(x=1) \\
4 \\
5 \\
6 \\
\text { Total }(x=0)\end{array}$ & $\begin{array}{c}\mathrm{L}_{102}=19 \\
\mathrm{~L}_{112}=23 \\
\mathrm{~L}_{122}=11 \\
\mathrm{~S}_{1 y \mathrm{k}} \\
\mathrm{L}_{\Sigma y \mathrm{k}} \\
\mathrm{L}_{002}=18 \\
\mathrm{~L}_{012}=9 \\
\mathrm{~L}_{022}=0 \\
\mathrm{SL}_{\Sigma y \mathrm{k}}\end{array}$ & $\begin{array}{c}2 / 3 \\
1 / 3 \\
0 / 3 \\
\\
B \\
0 / 3 \\
1 / 3 \\
2 / 3\end{array}$ & $\begin{array}{c}12.66 \\
7.66 \\
0 \\
20.33=R \\
B L_{\text {oyk }} \\
0 \\
3 \\
0 \\
3=S\end{array}$ & $\begin{array}{c}8.44 \\
2.55 \\
0 \\
10.99 \\
(B)^{2} \mathrm{~L}_{0 y \mathrm{k}} \\
0 \\
0.99 \\
0 \\
0.99\end{array}$ \\
\hline
\end{tabular}

Donde:

$\mathrm{L}_{1 y k}=$ Frecuencia de número de parejas por patrón de respuesta en el grupo de los casos expuestos.

$L_{\text {Oyk }}=$ Frecuencia de número de parejas por patrón de respuesta en el grupo de los casos no expuestos.

$\mathrm{K} \stackrel{\mathrm{yyk}}{=} \mathrm{N}$ úmero de controles por caso

$A=(k-y) /(k+1)$

$B=y /(k+1)$

$P_{1}=v(x)=$ Casos expuestos $/$ total de casos.

Razón de momios de Maentel-Haenszel

$O R_{M H}=R / S$

$R=\Sigma A L_{1 y k}$

$S=\Sigma B L_{0 y k}$

$\mathrm{V}_{1}\left(\right.$ varianza del $\left.O \mathrm{R}_{M H}\right)=\left(1 / \mathrm{R}^{2}\right)\left(\Sigma \mathrm{W}_{\mathrm{Ryk}}{ }^{2} \mathrm{~L}_{1 y \mathrm{k}}+0 \mathrm{R}^{2}{ }_{M H} \Sigma \mathrm{W}_{\text {Syk }}{ }^{2} \mathrm{~L}_{\text {Oyk }}\right)$.

IC $95 \%=\exp \left[\ln 0 R_{M H} \pm 1.96 ل_{V_{1}}\right]$

Fracción atribuible en los expuestos (FAE)

$F A E=1-1 / O R_{M H}$

$\mathrm{V}_{2}($ varianza de la $F A E)=(1-F A E)^{2} \mathrm{~V}_{1}$

IC $95 \%=$ FAE $\pm 1.96 V_{V_{2}}$

Fracción atribuible poblacional (FAP)

$F A P=v(x) F A E$

$\mathrm{V}_{3}$ (varianza de la prevalencia de exposición dentro de los casos $)=\{\mathrm{v}(\mathrm{x})[1-\mathrm{v}(\mathrm{x})]\} / \mathrm{N}$

IC $95 \%=v(x) \pm 1.96 ل v_{3}$

$\mathrm{V}_{4}($ varianza de la FAP $)=[\mathrm{V}(\mathrm{x})]^{2} \mathrm{~V}_{2}+(\mathrm{FAE})^{2} \mathrm{~V}_{3}+2 \mathrm{FAP}[(1-\mathrm{FAE}) / \mathrm{N}]$

IC $95 \%=$ FAP $\pm 1.96 \mathrm{VV}_{4}$ 